\title{
BMJ Open Cost-effectiveness of household contact investigation for detection of tuberculosis in Pakistan
}

Hamidah Hussain (DD , ${ }^{1,2}$ Amyn Malik (I) , ${ }^{2}$ Junaid F Ahmed, ${ }^{3}$ Sara Siddiqui, ${ }^{3}$
Farhana Amanullah, ${ }^{3}$ Jacob Creswell, ${ }^{5}$ Thorkild Tylleskär, ${ }^{1}$ Bjarne Robberstad ${ }^{1,6}$

To cite: Hussain $\mathrm{H}$, Malik $\mathrm{A}$, Ahmed JF, et al. Costeffectiveness of household contact investigation for detection of tuberculosis in Pakistan. BMJ Open 2021;11:e049658. doi:10.1136/ bmjopen-2021-049658

- Prepublication history for this paper is available online. To view these files, please visit the journal online (http://dx.doi. org/10.1136/bmjopen-2021049658).

Received 30 January 2021 Accepted 21 September 2021

Check for updates

(c) Author(s) (or their employer(s)) 2021. Re-use permitted under CC BY-NC. No commercial re-use. See rights and permissions. Published by BMJ.

${ }^{1}$ Centre for International Health, Department of Global Public Health and Primary Care, Universitetet i Bergen, Bergen, Norway

${ }^{2}$ Interactive Research and Development (IRD) Global, Singapore

${ }^{3}$ Global Health Directorate, Indus Health Network, Karachi, Pakistan

${ }^{4}$ The Indus Hospital, Karachi, Pakistan

${ }^{5}$ Stop TB Partnership, Geneva, Switzerland

${ }^{6}$ Section for Ethics and Health Economics, Department of Global Public Health and Primary Care, Universitetet i Bergen, Bergen, Norway

Correspondence to

Dr Hamidah Hussain;

hamidah.hussain@ird.global

\section{ABSTRACT}

Objectives Despite WHO guidelines recommending household contact investigation, and studies showing the impact of active screening, most tuberculosis (TB) programmes in resource-limited settings only carry out passive contact investigation. The cost of such strategies is often cited as barriers to their implementation. However, little data are available for the additional costs required to implement this strategy. We aimed to estimate the cost and cost-effectiveness of active contact investigation as compared with passive contact investigation in urban Pakistan.

Methods We estimated the cost-effectiveness of 'enhanced' (passive with follow-up) and 'active' (household visit) contact investigations compared with standard 'passive' contact investigation from providers and the programme's perspective using a simple decision tree. Costs were collected in Pakistan from a TB clinic performing passive contact investigation and from studies of active contact tracing interventions conducted. The effectiveness was based on the number of patients with TB identified among household contacts screened. Results The addition of enhanced contact investigation to the existing passive mode detected 3.8 times more cases of TB per index patient compared with passive contact investigation alone. The incremental cost was US\$30 per index patient, which yielded an incremental cost of US\$120 per incremental patient identified with TB. The active contact investigation was 1.5 times more effective than enhanced contact investigation with an incremental cost of US\$238 per incremental patient with TB identified. Conclusion Our results show that enhanced and active approaches to contact investigation effectively identify additional patients with TB among household contacts at a relatively modest cost. These strategies can be added to the passive contact investigation in a high burden setting to find the people with TB who are missed and meet the End TB strategy goals.

\section{BACKGROUND}

Tuberculosis (TB) remains a leading cause of morbidity and mortality, especially in low-income and middle-income countries. According to the latest estimates, 10 million people fell ill with TB in 2019, though only 7.1 million were reported to national programmes. Eight countries account for

\section{Strengths and limitations of this study}

The study was conducted in programmatic conditions in a high-prevalence setting.

- The study compares cost-effectiveness of three contact investigation approaches from health system perspective in a sequential approach.

- The data for the passive approach come from previous years and we were unable to account for any time trend.

- The study did not consider out-of-pocket expenditures for patients which underestimates the overall costs.

two-thirds of the reported TB burden in the world: India $(26 \%)$, Indonesia $(8.5 \%)$, China $(8.4 \%)$, the Philippines $(6 \%)$, Pakistan $(5.7 \%)$, Nigeria $(4.4 \%)$, Bangladesh $(3.6 \%)$ and South Africa $(3.6 \%) .{ }^{1-3}$ Reasons for the gap between estimated and notified individuals with TB include limited access to healthcare, poor diagnosis capacity for people who do access care, as well as under-reporting of people diagnosed. ${ }^{4-6}$

Undiagnosed people with TB continue to transmit TB to others. The risk of transmission is particularly high among members of households living with people with undiagnosed pulmonary TB. Studies have documented an infection rate of 30\%-50\% among household contacts of infectious adults, with the infection rate in children under 5 being as high as $72 \% .^{78}$ Of those infected with TB, $10 \%-20 \%$ develop the disease over their lifetimes, and this number is even higher for people who are immunocompromised, for example, when they are coinfected with HIV. $^{9-12}$

Household contact investigation is recommended as a means to address these challenges. ${ }^{13} 14$ In the light of the high infection rates, household contact investigation is a critical activity for TB programmes for two reasons. First, it allows early identification 
of additional household members who have TB disease and require immediate treatment, stopping transmission. Second, it allows programmes to identify people who can benefit from the treatment of TB infection to prevent disease progression, importantly children and people living with HIV. A meta-analysis of contact investigation showed that $3.1 \%$ of contacts in low-resource settings and $1.4 \%$ of contacts in high-resource settings have TB disease, making this a potential high yield strategy to find people with TB. ${ }^{1516}$ A large proportion of childhood TB can be identified through contact investigation, which is of particular value since global rates of detection among children are much lower than for adults. ${ }^{1}$

Household contact investigation can be carried out in many different ways along a continuum of passive and active approaches. ${ }^{17}$ In passive contact tracing, the index patient is asked to bring in their family members for screening to the facility, while in active contact tracing, healthcare workers visit the index patient's home. Between the active and passive modes, lies the 'enhanced' form of contact tracing, in which health workers make reminder phone calls and follow-up with the family and encourages them to come to the facility for screening.

Despite WHO guidelines recommending household contact investigation ${ }^{18} 19$ and studies documenting the outcomes of active approaches most TB programmes in resource-limited settings only carry out passive contact investigation and even then, implementation is limited..$^{2021}$ A cluster randomised controlled trial demonstrated that contact investigation plus passive case finding (PCF) was beneficial compared with PCF alone. ${ }^{22}$ The cost of active contact investigation, including additional efforts required by already stretched healthcare providers, has often been cited as a barrier to its implementation. ${ }^{23}$ However, little data are available for the additional cost of implementing active contact investigation, and especially so when implemented under routine programme conditions. A study from Malaysia reported the cost of active contact investigation to be US $\$ 6.60$ per a single contact tracing visit with a yield of $0.5 \% .{ }^{24}$ In Peru, adding active contact tracing to PCF incurred an incremental cost of US $\$ 48.8$ to evaluate household contacts of an index TB patient, with an incremental cost-effectiveness ratio (ICER) of US $\$ 1811$ per Disability Adjusted Life Years (DALY) averted. ${ }^{25}$ We were not able to identify studies reporting costs or cost-effectiveness for the enhanced mode of contact tracing.

The objective of this study was to estimate the cost and cost-effectiveness of the enhanced and active contact tracing interventions in a high-burden programmatic setting, compared with the existing passive approach.

\section{METHODS}

\section{Setting}

The costing study was a subset of a larger study where an active case finding intervention was implemented for children with TB. The study was conducted at four TB treatment and reporting centres in Kotri, a rural town in Sindh, Pakistan. All children presenting to these facilities were verbally screened for symptoms of $\mathrm{TB}$ and those considered to be at high risk of having TB were further investigated. Children diagnosed with TB were started on treatment, and we conducted contact investigation for their household contacts. ${ }^{26}$ The household contact investigation reported here was carried out at one of the four centres (Institute of Chest Disease Hospital) from April 2015 to March 2016.

\section{Interventions}

For the study, the index patient was the first person identified with TB in the family, while household contacts were defined as people living in the same household as the index patient.

As part of the routine programme passive contact investigation is conducted where, index patients are counselled to bring their household contacts for evaluation. Contacts who respond are evaluated for TB symptoms and risk factors. A limitation of this approach is that household contacts screened were recorded only as a TB contact and not linked directly to the index TB patient in a specific household. There is no routine follow-up to see if the specific contacts attended the facility for screening or not.

As part of the intervention we instituted enhanced contact investigation as an additional step which included follow-up with the families for contact investigation. Adults and guardians of children under 15 years of age newly diagnosed with TB were asked about the presence of TB symptoms or household members on TB treatment in their families. They were counselled to bring their enumerated household contacts for evaluation, as in the passive approach. If the enumerated household contacts did not come to the facility for assessment after 1 week, a reminder phone call was made, followed by second phone call after another week. If the family still had not come 2 weeks after the second phone reminder, active contact investigation was implemented. Active contact investigation included health workers conducting a household visit to verbally screen the family at home and to counsel the family to go to the clinic for further evaluation (figure 1).

At the clinic, enumerated household contacts were screened by existing TB doctors in the passive system or by trained health workers for enhanced and active contact investigation. All contacts were verbally screened for symptoms of TB such as cough of more than 2 weeks, contact with someone other than the index patient who had TB, glandular swelling, fever lasting more than 2 weeks, night sweats and inappropriate weight loss. Individuals with suggestive symptoms or additional exposure were referred to the project's medical officer for further evaluation. They then received a chest X-ray and were asked to provide a sputum sample for smear microscopy. A complete blood count and erythrocyte sedimentation rate was done for child contacts to aid in diagnosis as 


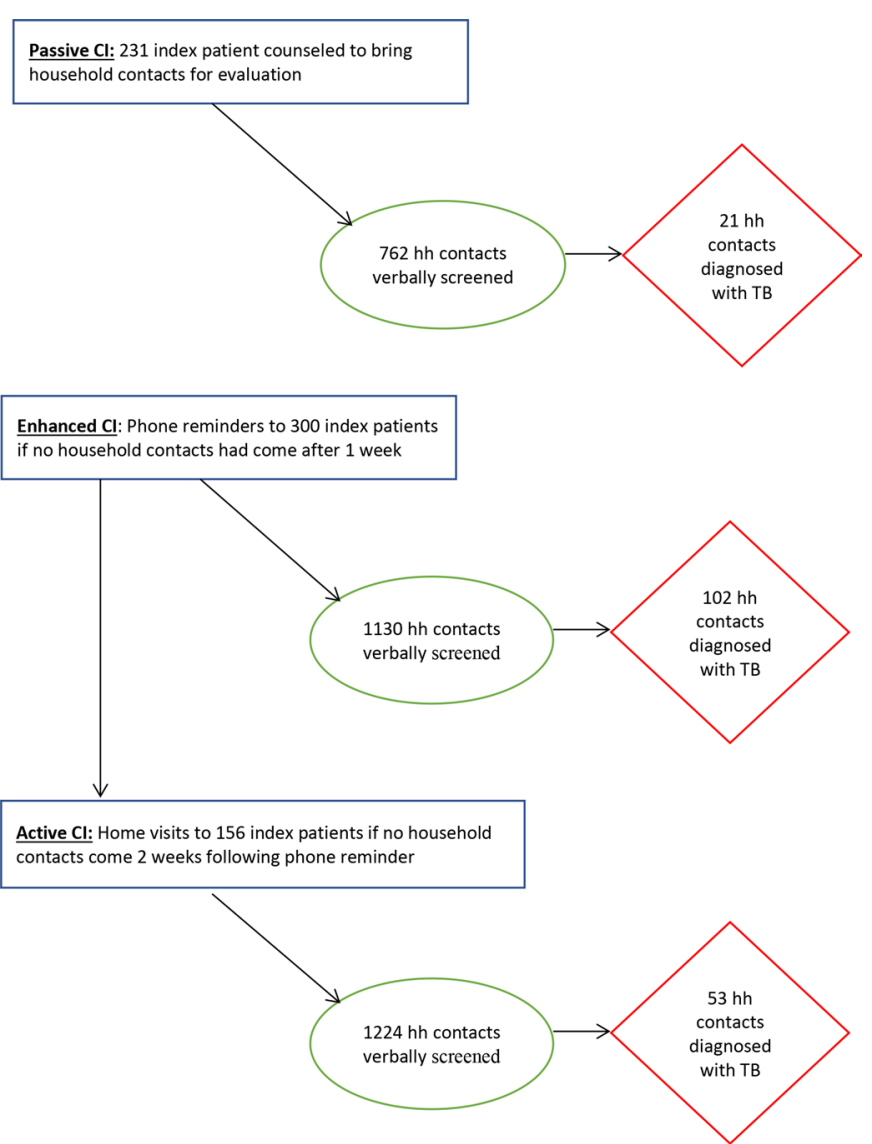

Figure 1 Household contacts evaluated and diagnosed for TB by passive, enhanced and active contact investigation interventions $(\mathrm{Cl})$. TB, tuberculosis.

indicated. Contacts diagnosed with TB were started on TB treatment in line with the National TB Programme (NTP) guidelines. Children under 5 years of age in whom TB disease was ruled out were offered isoniazid preventive therapy as per NTP guidelines. ${ }^{26}$ All clinical evaluations and investigations were provided without any charge to the contacts.

\section{Data collection}

For the passive approach, health facility staff recorded data using a paper-based system which were then abstracted for the study. For enhanced and active contact interventions project based trained community health workers and doctors administered questions to assess TB symptoms/ risks and documented results of clinical evaluation and diagnostic tests using a custom-built smart phone-based data collection application with built-in decision support developed for the project. $^{26}$

\section{Cost parameters}

Costs for this systematic contact tracing activities were collected from the perspective of the operational programme and the health facility and included recurrent and capital cost items. As capital costs for the building were not available, we approximated rent and utilities of running a similar structure, and we used these in place of the capital costs.
We identified cost items and quantified resource use for all activities related to contact investigation. They included personnel, diagnostic test, supervision and monitoring by facility or project staff and communications. For the passive system, cost information was obtained from the health facility accounting system. We identified one physician and one health worker who were involved in the existing passive system at the TB clinic. We estimated their time spent on evaluation of household contacts through expert opinion and allocated salaries proportionate to this time as compared with other activities. Unit costs for TB diagnostic tests, chest X-rays and smear microscopy were as billed to the project by the health facility. Costs for diagnostic tests were estimated by multiplying their unit costs with the number of people tested. As communications, supervision and training costs for the existing passive programme were not available through the facility records, we assumed the same costs as incurred by the enhanced contact investigation intervention. At the TB clinic, data were collected on paper-based systems and the costs for registers and forms are reported with stationary.

For the additional costs of performing enhanced and active contact investigations, data were extracted from the project accounting system. One full-time health worker was recruited for enhanced intervention while the active contact investigation required three additional health workers. A fixed amount of travel costs for home visits was built into the salary for health workers. For all other personnel such as physician, field supervisor and programme coordinators, time spent on the contact investigation intervention was estimated using an activitybased costing methodology and costs were allocated according to the proportion of time spent on the intervention relative to other activities. ${ }^{27} 28$ Cost of diagnostic investigations per person screened (chest X-ray, smear microscopy and complete blood), communications (data and phone), training and stationery were as incurred. The cost for the development of electronic data capture was allocated based on the number of patients screened in each intervention, while the cost of phones and laptops used to capture data were allocated as per the personnel time that used them. We annuitised these capital costs over a period of 3 years using a $3 \%$ discount rate. ${ }^{29}{ }^{30}$ Costs were collected in Pakistan rupees and converted to US dollars using the average exchange rate for the years 2015 and 2016 (US\$1=PKR103.1).

\section{Effectiveness of contact investigation}

The effectiveness of the contact investigation procedure was evaluated based on the number of people with TB identified per household screened after verbal screening and diagnostic tests. Our study was divided into baseline and intervention periods. Historical data for passive contact investigation were used as the comparator. In the year preceding the intervention, the passive approach screened 762 contacts from a total of 231 index patient households to identify 21 people with TB during this 


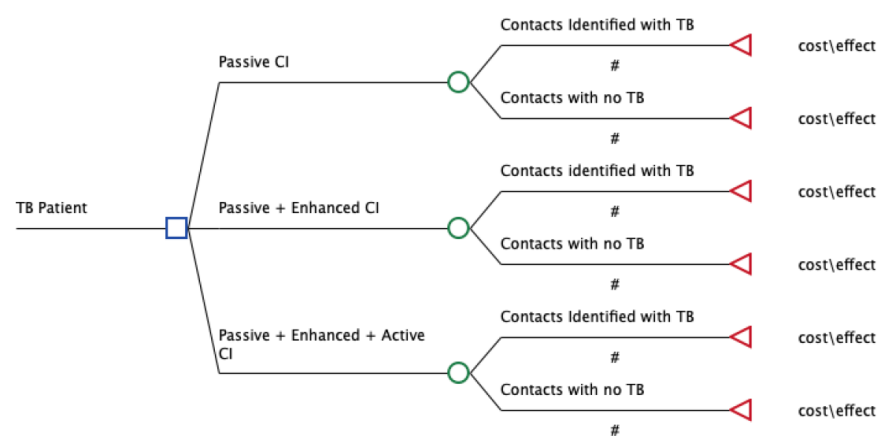

Figure 2 Decision tree for household contacts evaluated for TB by passive, enhanced and active contact investigation interventions $(\mathrm{Cl})$. TB, tuberculosis.

baseline period (figure 1). During the intervention period, enhanced and active contact investigation were implemented, and contacts from 300 households were evaluated. Of these, 1130 people from 144 families came to the health facility after phone reminders (enhanced) and 102 were diagnosed with TB. When home visits were conducted for 156 households that failed to respond to the enhanced strategy (active), we evaluated 1224 people and identified 53 additional people with $\mathrm{TB}$ disease (figure 1).

\section{Decision model and analysis}

A simple decision tree was created in TreeAge Pro 2020 (TreeAge Pro, Williamston, Massachusetts, USA) to estimate the cost-effectiveness of enhanced and active contact investigation compared with passive contact investigation. The decision tree includes the three alternatives for contact investigation; (1) Passive, (2) Passive +Enhanced and (3) Passive +Enhanced +Active (figure 2). The three intervention alternatives represent different levels of intensity of contact investigation and are considered to be mutually exclusive. The more intense alternatives are more expensive than the less intensive ones, but also represent new possibilities for identifying contacts with TB (table 1). The results are presented as absolute and incremental costs and patients with TB identified, and ICERs between the alternatives.

We conducted probabilistic sensitivity analyses using Monte Carlo simulations with 10000 iterations to explore the effects of combined uncertainties in key parameters. Gamma distributions were used for cost parameters, and beta distributions for the probability of patients with $\mathrm{TB}$ per family screened.$^{31}$ For sensitivity analyses, upper and lower values were defined for each parameter as mean values $\pm 20 \%$.

\section{RESULTS}

In the enhanced and active contact tracing, 2354 household contacts from 300 index patients were screened, of whom $49 \%$ were children less than 15 years of age, and $45 \%$ were female. The mean age for child contacts was 6.4 years (SD 3.7, IQR: $3-9$ ), and 54\% were males and the mean age for adult contacts was 33 years (SD 13.4, IQR: 21-41) and 53\% were males. The enhanced contact investigation intervention was able to find 2.45 times (95\% CI: 1.52 to 4.14 ) more people with TB than the passive programme when it was implemented. While the active intervention implemented 3 weeks following the index patient counselling identified 2.11 times (95\% CI: 1.33 to 3.52 ) more people with TB compared with passive contact investigation.

Overall, the passive programme incurred US\$10 659 over 1 year and it cost US $\$ 46$ per household screened with TB. The enhanced contact investigation incurred an additional US\$30 to screen a household with an overall addition of US $\$ 8938$ to the yearly programme cost. Of the additional costs, human resources $(42 \%)$ and electronic data collection (24\%) were the most significant cost drivers. Active contact investigation incurred an additional US $\$ 42$ per household screened for TB above the enhanced model, and the programme cost a further US\$12 685 to the enhanced contact investigation of which human resources $(57 \%)$ and electronic data capture (18\%) were the largest components. (table 2)

The passive +enhanced contact investigation of one index patient was 3.8 times more effective than passive contact investigation alone, increasing absolute case detection rate from 0.09 to 0.34 . The incremental cost was US $\$ 30$ per index patient, which yielded an incremental cost of US $\$ 120$ per incremental patient identified with TB. While the passive +enhanced+active contact investigation of one index patient was 1.5 times more effective than enhanced contact investigation with an incremental cost of US $\$ 238$ per incremental patient with TB identified as compared with the baseline passive approach (table 3).

The cost-effectiveness acceptability curves illustrate the probabilities that each intervention is cost-effective

Table 1 Modelling inputs, assumptions and ranges for passive, enhanced and active contact investigation (Cl)

\begin{tabular}{|c|c|c|c|c|c|}
\hline Interventions & $\begin{array}{l}\text { Total cost } \\
\text { (US\$) }\end{array}$ & $\begin{array}{l}\text { Index } \\
\text { patient } \\
\text { with TB }\end{array}$ & $\begin{array}{l}\text { Total contacts } \\
\text { diagnosed with TB } \\
\text { (lower and upper } \\
\text { limit) }\end{array}$ & $\begin{array}{l}\text { Cost per index } \\
\text { TB patient family } \\
\text { screened } \\
\text { (US\$) }\end{array}$ & $\begin{array}{l}\text { Probability of finding } \\
\text { a patient with TB per } \\
\text { household screened } \\
\text { (lower and upper limit) }\end{array}$ \\
\hline Passive +enhanced Cl & US\$19597 & 300 & $102(82-122)$ & $76(61-91)$ & $0.34(0.27-0.40)$ \\
\hline Passive +enhanced + active $\mathrm{Cl}$ & US\$32282 & 300 & $155(124-186)$ & $118(94-142)$ & $0.52(0.41-0.62)$ \\
\hline
\end{tabular}

TB, tuberculosis. 
Table 2 Cost (USD) of household contact screening for passive, enhanced and active contact investigation activities (upper panel), and cumulative costs per intervention arm (lower panel)

\begin{tabular}{|c|c|c|c|}
\hline $\begin{array}{l}\text { Intervention activities } \\
\text { Cost categories }\end{array}$ & $\begin{array}{l}\text { Passive contact } \\
\text { investigation } \mathrm{N}=231(\%)\end{array}$ & $\begin{array}{l}\text { Enhanced contact } \\
\text { investigation } \mathrm{N}=300(\%)\end{array}$ & $\begin{array}{l}\text { Active contact } \\
\text { investigation } \mathrm{N}=300(\%)\end{array}$ \\
\hline \multicolumn{4}{|l|}{ Recurrent costs: } \\
\hline Personnel & $5354(50)$ & $3835(42)$ & $7348(57)$ \\
\hline Diagnostic tests & $1478(14)$ & $2192(24)$ & $2374(18)$ \\
\hline Communication & $58(1)$ & $204(2)$ & $204(2)$ \\
\hline Training & $72(1)$ & $72(1)$ & $70(1)$ \\
\hline Stationary & $88(1)$ & $22(0)$ & $24(0)$ \\
\hline Subtotal recurrent costs & 10659 & 6520 & 10096 \\
\hline \multicolumn{4}{|l|}{ Capital costs: } \\
\hline Annuitised capital costs ( $3 \%$ discount rate) & & 2419 & 2589 \\
\hline Total costs per activity & 10659 & 8938 & 12685 \\
\hline Total costs per activity per index patient & 46 & 30 & 42 \\
\hline Intervention arm & Passive & Passive +enhanced & $\begin{array}{l}\text { Passive +enhanced }+ \\
\text { active }\end{array}$ \\
\hline $\begin{array}{l}\text { Total cumulated costs per index patient per } \\
\text { arm for household contacts evaluated for TB }\end{array}$ & 46 & 76 & 118 \\
\hline No of contacts diagnosed with TB & 21 & 123 & 176 \\
\hline
\end{tabular}

for a range of willingness to pay for health when taking the combined parameter uncertainty into account. The enhanced strategy becomes optimal if the willingness to pay exceeds US $\$ 120$ per additional patient with TB that is identified. If willingness to pay exceeds about US $\$ 238$ per TB case identified, ${ }^{32}$ the active contact investigation has the highest probability of being cost-effective of the three alternatives (figure 3).

One-way sensitivity analyses were conducted to explore the impact of uncertainties in single model parameters. These are represented in a tornado diagram in the decreasing order of the parameters' potential influence on the ICER (figure 4). As the passive contact investigation is standard we plotted the tornado diagram for enhanced versus active contact investigation strategies. Cost and effect parameters were varied over a predetermined range (table 1). The ICER was most sensitive to the probability of identifying a patient through active case finding, and ranged between US $\$ 150$ and US $\$ 600$ per case detected when probabilities were varied between 0.62 and 0.41 , respectively.

\section{DISCUSSION}

The enhanced contact investigation strategy, in combination with the passive system, was 3.8 times more likely to identify patients with TB among household contacts than the passive contact investigation alone. The addition of household visits further improved case detection and may be necessary if we are to achieve the End TB strategy goals. ${ }^{33}$ Unsurprisingly, both the enhanced and active strategies require more resources

Table 3 Incremental cost-effectiveness of household contact screening for passive, enhanced and active contact investigation interventions from the TB programme perspective

\begin{tabular}{lcccc}
\hline Strategy & $\begin{array}{l}\text { Cost per } \\
\text { strategy }\end{array}$ & $\begin{array}{l}\text { Incremental } \\
\text { cost }\end{array}$ & Effect & $\begin{array}{l}\text { Incremental } \\
\text { effect }\end{array}$ \\
\hline Passive contact investigation & 46 & & 0.09 & \\
Passive +enhanced contact investigation & 76 & 30 & 0.34 & 0.25 \\
Passive +enhanced+active contact investigation & 118 & 42 & 0.52 & 0.18 \\
\hline
\end{tabular}

ICER, incremental cost-effectiveness ratio; TB, tuberculosis. 


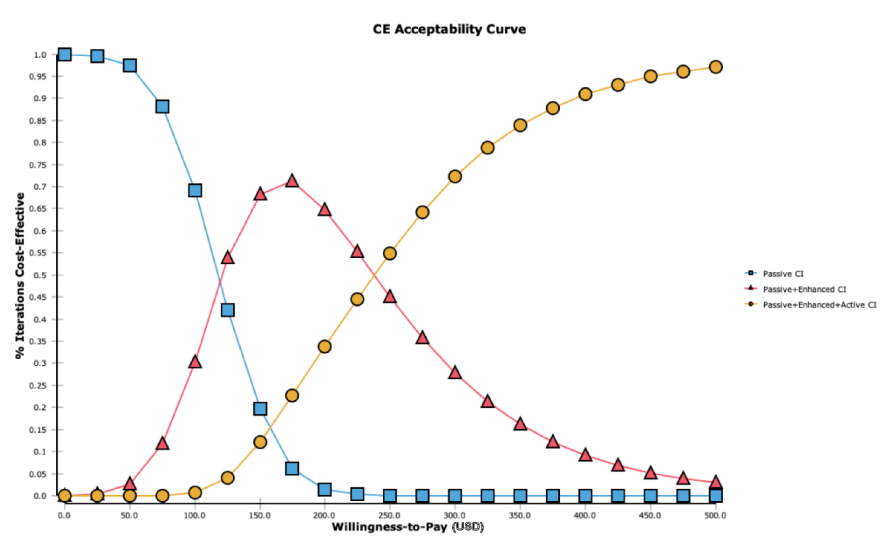

Figure 3 Cost-effectiveness (CE) acceptability curves for passive, enhanced and active contact investigation interventions $(\mathrm{Cl})$ for a range of willingness to pay per household screened.

than the existing passive scheme, and the additional benefits must therefore be weighed against their additional costs, but increased performance and output requires more funding for impactful interventions. ${ }^{34}$

Studies and systematic reviews have documented that enhanced or active household contact investigation has been able to find more people with TB compared with PCF. $^{12131735}$ These studies further conclude that improved case detection is cost-effective compared with the passive approach. Contact investigation can be conducted in a myriad of ways and using different algorithms and approaches. ${ }^{17}$ Many programmes opt for a more passive approach due to the ease of implementation and lower costs. However, there have been no studies we could identify that have compared different modalities of contact investigation to each other. The WHO guidelines identify that comparisons of different types of contact investigation is a current knowledge gap and our findings aid this void and should be followed by additional studies with costing analyses.

In low-burden countries, contact investigation is a requirement for a TB programme to be effective. ${ }^{36}$ According to the US Centers for Disease Control and

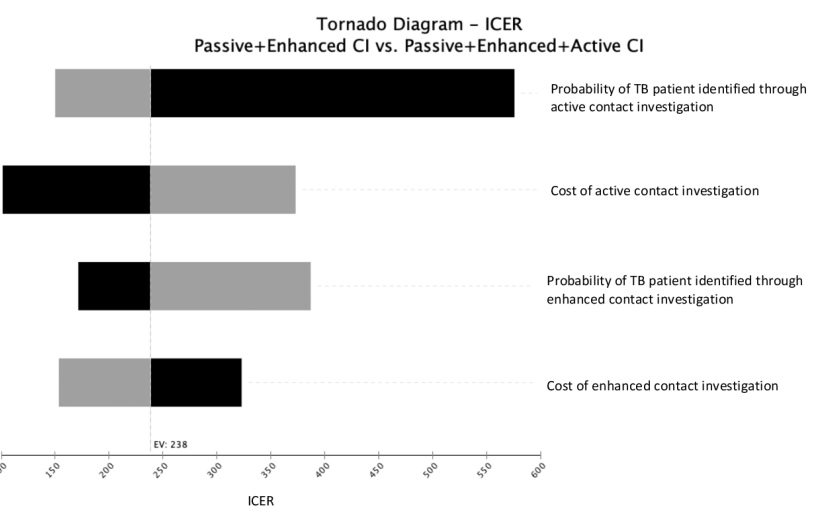

Figure 4 One-way sensitivity analyses for the ICERs of enhanced contact investigation compared with active contact investigation (Cl). ICER, incremental cost-effectiveness ratio; TB, tuberculosis.
Prevention, it played an essential role in decreasing TB incidence by $44 \%$ in the USA. ${ }^{37}$ A meta-analysis suggests that $\mathrm{TB}$ contact investigation should be considered to improve early TB case detection and decrease transmission in high-incidence areas as well. ${ }^{13}$ However, in low-income and middle-income countries, contact investigation has been viewed as expensive and, therefore, a low priority. Programmes do not undertake TB contact investigation as they have limited human resources. This project added health workers to support phone calls, counselling and home visits, which led to an increase in costs. However, these are necessary costs if we are to reach all people with TB. With contact investigation, people with TB are diagnosed early and initiated on treatment, which benefits the broader community by reducing continuing transmission. ${ }^{38}$ These benefits of future TB cases prevented over time are not captured by the current analytical model, and in this regard, our results can be considered to be conservative. If contact investigation interventions result in earlier detection of household contacts with active $\mathrm{TB}$, this programme would reduce the spread of $\mathrm{TB}$ in the community even more effectively and be even more cost-effective.

Our study is subject to limitations. We initiated the enhanced intervention if the family did not come to the clinic for evaluation within 1 week of a patient with TB diagnosed and initiated on treatment. The passive system, if given more time than 1 week may potentially have had a larger yield. However, the historical data show that the number of people identified by the passive system during the implementation phase was similar to what we estimated in the baseline survey. Second, we only consider people with TB detected and did not consider outcomes of subsequent treatment in this analytical model. But in the larger project, in which this study was embedded, $98 \%$ of children diagnosed with TB were started on treatment and had over $94 \%$ treatment success rate. ${ }^{26} 39$ Third, out-ofpocket expenditures for patients was not considered. Costs such as transportation to the health facilities for evaluation, cost of diagnostic tests and loss of work time may be potential barriers for the majority of the TB affected families coming to the health facilities for evaluation. Arrangements for transportation of contacts to health facilities for diagnostic tests and transportation of sputum specimens for examination should be included in the national policy to increase the detection of patients with TB. Lastly, the household with child TB is likely to have transmission with in the household making contact investigation efficient and cost-effective in these contacts, but it may not be generalisable in household with an adult patients with TB. Future research may consider to conduct similar costing studies in the urban areas as the cost may be different than the rural setting in which our study was based and to integrate data on the overall economic burden to households that can be averted with an active TB contact investigation programme. In 
addition, the study was conducted at only one centre, and other health facilities with different levels of preexisting capacity and infrastructure may yield different cost estimates.

An important strength of the study is that it was performed alongside implementation in district TB clinics in a high-prevalence setting. Data were, therefore, collected prospectively in a programmatic setting. A robust monitoring and evaluation system was put in place, and the District TB Control officers verified all notifications as would have been done in routine scale up.

\section{CONCLUSION}

Our results show that active approaches to contact investigation identify more people with $\mathrm{TB}$ among household contacts at a relatively modest cost addressing an identified global knowledge gap. These strategies can be added to passive contact investigation approaches in a high burden setting to find the missing patients with TB and meet the End TB strategy goals.

Twitter Amyn Malik @amynmalik and Jacob Creswell @Jacob_Creswell

Contributors Conception: HH, AM and FA. Data collection: HH, AM, SS and FA. Analysis: HH, AM and BR. Writing: HH, AM, JFA, SS, FA, JC, TT and BR. HH accepts all responsibility for the work and/or the conduct of the study, had access to the data, and controlled the decision to publish.

Funding Active case finding from October 2014 to March 2016 was supported through Stop TB Partnership's TB REACH initiative. TB REACH is generously supported by Global Affairs Canada. Active case finding from October 2016 to March 2018 was supported through The Global Fund. Award/Grant number is not applicable.

\section{Competing interests None declared.}

Patient and public involvement Patients and/or the public were not involved in the design, or conduct, or reporting, or dissemination plans of this research.

Patient consent for publication Not applicable.

Ethics approval This study was approved by the Institutional Review Board (IRB) of Interactive Research and Development (IRD), OHRP Registration No. 00005148.

Provenance and peer review Not commissioned; externally peer reviewed.

Data availability statement Data are available from the corresponding author on reasonable request. Data are available from the corresponding author upon reasonable request.

Open access This is an open access article distributed in accordance with the Creative Commons Attribution Non Commercial (CC BY-NC 4.0) license, which permits others to distribute, remix, adapt, build upon this work non-commercially, and license their derivative works on different terms, provided the original work is properly cited, appropriate credit is given, any changes made indicated, and the use is non-commercial. See: http://creativecommons.org/licenses/by-nc/4.0/.

\section{ORCID iDs}

Hamidah Hussain http://orcid.org/0000-0002-6585-9815

Amyn Malik http://orcid.org/0000-0003-4875-9916

\section{REFERENCES}

1 The World Health Organization. Global tuberculosis report 2019. Geneva: World Health Organization, 2019.

2 World Health Organization. Global tuberculosis report 2020. Geneva: World Health Organization, 2020.

3 The Global Fund. Tuberculosis, 2018. Available: https://www. theglobalfund.org/en/tuberculosis/ [Accessed 15 Nov 2020].
4 Vyas A, Creswell J, Codlin AJ, et al. Community-based active casefinding to reach the most vulnerable: tuberculosis in tribal areas of India. Int J Tuberc Lung Dis 2019;23:750-5.

5 Claassens MM, Jacobs E, Cyster E, et al. Tuberculosis cases missed in primary health care facilities: should we redefine case finding? Int $J$ Tuberc Lung Dis 2013;17:608-14.

6 MacPherson P, Houben RMGJ, Glynn JR, et al. Pre-Treatment loss to follow-up in tuberculosis patients in low- and lower-middle-income countries and high-burden countries: a systematic review and metaanalysis. Bull World Health Organ 2014;92:126-38.

7 Davies PD. The natural history of tuberculosis in children. A study of child contacts in the Brompton Hospital child contact clinic from 1930 to 1952 . Tubercle 1961;42(Suppl:1-40.

8 Starke JR, Jacobs RF, Jereb J. Resurgence of tuberculosis in children. J Pediatr 1992;120:839-55.

9 Bloom BR, Murray CJ. Tuberculosis: commentary on a reemergent killer. Science 1992;257:1055-64.

10 Vynnycky E, Fine PE. Lifetime risks, incubation period, and serial interval of tuberculosis. Am J Epidemiol 2000;152:247-63.

11 Devadatta S, Dawson JJ, Fox W, et al. Attack rate of tuberculosis in a 5 -year period among close family contacts of tuberculous patients under domiciliary treatment with isoniazid plus PAS or isoniazid alone. Bull World Health Organ 1970;42:337-51.

12 Schaaf HS, Gie RP, Kennedy M, et al. Evaluation of young children in contact with adult multidrug-resistant pulmonary tuberculosis: a 30-month follow-up. Pediatrics 2002;109:765-71.

13 World Health Organization. Recommendations for investigating contacts of persons with infectious tuberculosis in low- and middleincome countries. Geneva, Switzerland: World Health Organization, 2012.

14 Morrison J, Pai M, Hopewell PC. Tuberculosis and latent tuberculosis infection in close contacts of people with pulmonary tuberculosis in low-income and middle-income countries: a systematic review and meta-analysis. Lancet Infect Dis 2008;8:359-68.

15 Fox GJ, Barry SE, Britton WJ, et al. Contact investigation for tuberculosis: a systematic review and meta-analysis. Eur Respir $J$ 2013;41:140-56.

16 Fox GJ, Nhung NV, Sy DN, et al. Household contact investigation for tuberculosis in Vietnam: study protocol for a cluster randomized controlled trial. Trials 2013;14:342.

17 Blok L, Sahu S, Creswell J, et al. Comparative meta-analysis of tuberculosis contact investigation interventions in eleven high burden countries. PLoS One 2015;10:e0119822.

18 World Health Organization. Recommendations for investigating contacts of persons with infectious tuberculosis in low- and middle-income countries. Geneva: World Health Organization, 2012.

19 World Health Organization. Systematic screening for active tuberculosis: principles and recommendations. Geneva: World Health Organization, 2013.

20 Hwang TJ, Ottmani S, Uplekar M. A rapid assessment of prevailing policies on tuberculosis contact investigation. Int J Tuberc Lung Dis 2011;15:1620-3.

21 Shah SA, Qayyum S, Abro R, et al. Active contact investigation and treatment support: an integrated approach in rural and urban Sindh, Pakistan. Int J Tuberc Lung Dis 2013;17:1569-74.

22 Fox GJ, Nhung NV, Sy DN, et al. Household-Contact investigation for detection of tuberculosis in Vietnam. N Engl J Med Overseas Ed 2018;378:221-9.

23 Tesfaye L, Lemu YK, Tareke KG, et al. Exploration of barriers and facilitators to household contact tracing of index tuberculosis cases in Anlemo district, Hadiya zone, southern Ethiopia: qualitative study. PLoS One 2020;15:e0233358.

24 Atif M, Sulaiman SAS, Shafie AA, et al. Tracing contacts of TB patients in Malaysia: costs and practicality. Springerplus 2012;1:40.

25 Shah L, Rojas M, Mori O, et al. Cost-Effectiveness of active casefinding of household contacts of pulmonary tuberculosis patients in a low HIV, tuberculosis-endemic urban area of lima, Peru. Epidemiol Infect 2017;145:1107-17.

26 Malik AA, Amanullah F, Codlin AJ, et al. Improving childhood tuberculosis detection and treatment through facility-based screening in rural Pakistan. Int J Tuberc Lung Dis 2018;22:851-7.

27 Waters $\mathrm{H}$, Abdallah $\mathrm{H}$, Santillán D. Application of activity-based costing (ABC) for a Peruvian NGO healthcare provider. Int $J$ Health Plann Manage 2001;16:3-18.

28 Hussain $\mathrm{H}$, Waters $\mathrm{H}$, Omer SB, et al. The cost of treatment for child pneumonias and meningitis in the Northern areas of Pakistan. Int $J$ Health Plann Manage 2006;21:229-38.

29 Bergmo TS. How to measure costs and benefits of eHealth interventions: an overview of methods and frameworks. J Med Internet Res 2015;17:e254. 
30 Drummond M, Sculpher M, Torrance G, et al. Methods for the economic evaluation of health care programmes. 3rd edition. Oxford, UK: Oxford University Press, 2005.

31 Gray AM, Clarke PM, Wolstenholme JL, et al. Applied methods of cost-effectiveness analysis in healthcare. United States: Oxford University Press, 2010.

32 Shillcutt SD, Walker DG, Goodman CA, et al. Cost effectiveness in low- and middle-income countries: a review of the debates surrounding decision rules. Pharmacoeconomics 2009;27:903-17.

33 The World Health Organization. The end TB strategy, global strategy and targets for tuberculosis prevention, care and control after 2015. World Health Organization, 2018.

34 Stop TB Partnership. UN high-level meeting on TB key targets \& commitments for 2022. Geneva, Switzerland: Stop TB Partnership, 2018.
35 Htet KKK, Liabsuetrakul T, Thein S. Cost-effectiveness of a new strategy to detect pulmonary tuberculosis in household contacts in Myanmar. Int J Tuberc Lung Dis 2017;21:181-7.

36 Joint Tuberculosis Committee of the British Thoracic Society. Contro and prevention of tuberculosis in the United Kingdom: code of practice 2000. Thorax 2000;55:887-901.

37 Taylor Z, Nolan CM, Blumberg HM, et al. Controlling tuberculosis in the United States. recommendations from the American thoracic Society, CDC, and the infectious diseases Society of America. MMWR Recomm Rep 2005;54:1-81.

38 Dowdy DW, Basu S, Andrews JR. Is passive diagnosis enough? The impact of subclinical disease on diagnostic strategies for tuberculosis. Am J Respir Crit Care Med 2013;187:543-51.

39 Malik AA, Amanullah F, Jaswal M, et al. High yields from contact investigation of child index TB patients. Int J Tuberc Lung Dis 2020;24:850-2 